\title{
PERFORMANCE MEASURES IN THE GENETIC DESIGN OF DIGITAL CONTROLLERS FOR ROBOTIC MANIPULATORS
}

\author{
B Porter and C Allaoui \\ Intelligent Machinery Division \\ Research Institute for Design, Manufacture, and Marketing \\ University of Salford \\ Salford M5 4WT \\ England
}

\section{INTRODUCTION}

It was shown by Porter and Abidin [4] that the design of digital trajectory-tracking controllers for robotic manipulators can be readily effected by using the methodologies of Porter et al [7] for the design of fastsampling error-actuated digital multivariable PID controllers. These design methodologies are characterised by the following very important features:
\end{abstract}

ABSTRACT

Genetic algorithms are used to design digital multivariable PID controllers for robotic manipulators for typical trajectory-tracking tasks when various different performance measures are used. It is thus shown that, by using an appropriate performance measure, the set of controller parameters can be readily found that determines the optimal time-domain trajectory-tracking behaviour for such tasks. This use of genetic algorithms is illustrated by the design of digital trajectory-tracking PID controllers for a typical three-degree-of-freedom robotic manipulator.

1) the design equations for the controller matrices are directly expressed in terms of the step-response matrices of the plants under control;

2) the robustness characteristics of the controllers are simply expressed in terms of the step-response matrices of the nominal and actual plants.

However, although these robustness results of Porter and Abidin [4] greatly facilitate the design of digital trajectorytracking controllers for robotic manipulators, these theoretical results are valid only asymptotically (i.e., as the frequency of the digital PID controller becomes infinite). Since it has so far proved impossible to obtain theoretical non-asymptotic robustness results for digital multivariable PID controllers with finite sampling frequencies, genetic algorithms [2] [1] were used by Porter and Allaoui [5] to robustify such controllers for robotic manipulators performing typical trajectory-tracking tasks. This process of robustification was effected by using genetic algorithms to determine the optimal quadruple of controller turning parameters for such trajectory-tracking tasks (which include sudden changes in payload). These genetic design results of Porter and Allaoui [5] are the natural extension to robotic control problems of the non-robotic results of Porter and Jones [6] and Porter, Mohamed, and Jones [8].

In the genetic design procedure of Porter and Allaoui [5], the minimum integral error norm for any trajectory-tracking task was regarded as the ultimate design requirement; but it is important to note that other performance measures can be used equally well in the context of genetic design. In this paper, a detailed comparison is therefore made of the performance of genetically designed trajectory-tracking controllers for robotic manipulators when the following three performance measures are used:

1) the integral error norm;

2) the weighted sum of the integral error norm and the integral error velocity norm;

3) the weighted sum of the integral error norm and the integral control velocity norm.

These general results are illustrated by the genetic design of robustified digital trajectory -tracking PID controllers for the three-degreeof-freedom robotic manipulator previously investigated by Porter and Allaoui [5]. 


\section{GENETIC DESIGN PROCEDURE}

The methodology of Porter et al [7] for the design of error-actuated digital PID controllers relates to linearised multivariable plants governed on the continuous-time set $T=[0,+\infty)$ by state and output equations of the respective forms

and

$$
\dot{x}(t)=A x(t)+B u(t)
$$

$$
y(t)=C x(t)
$$

In equations (1) and (2). $x(t) \in R^{n}, u(t) \Subset R^{l}, y(t) \in R^{l}, A \in R^{n x n}, B \in R^{n x t}$, a $n d$ $C e R^{b m}$. In addition, it is assumed that the plants under control are llike most robotic manipulators) completely irregular so that

$$
\operatorname{rank} C B=0
$$

and

$$
\text { rank } C A B=l
$$

The digital PID controllers for such plants are governed on the discrete-time set $T_{T}=\{0, T, 2 T, \ldots, k T, \ldots\}$ by control-law equations of the form

$$
u(k T)=K_{1} r(k T)+K_{2} z(k T)
$$

In equation (5), $T \in R^{+}$is the sampling period, $K_{1} \notin R^{l x l}$ and $K_{2} \in R^{l x l}$ are the proportional and integral controller matrices, and the vectors $r(k I) \in R^{l}$ and $z(k T) \in R^{l}$ are generated in accordance with the difference equations

$$
\begin{array}{r}
s\{(k+1) T\}=-\alpha s(k T)+e(k T), \text { (6a) } \\
r(k T)=-\frac{2}{T}(1+\alpha) D s(k T)+\left(I_{l}+\frac{2}{T} D\right) e(k T),(6 \mathrm{~b})
\end{array}
$$

and

$$
z\{(k+1) T\}=z(k T)+\operatorname{Tr}(k T) \quad \text { (7) }
$$

Moreover, in equation $(6), \alpha \in(-1,+1)$, $s(k T) \in R^{l}, e(k T)=v(k T)-y(k T) \in R^{l}$ is the error vector, $v(k T) \in R^{l}$ is the set-point command vector, and the derivative matrix $D \in R^{l x l}$ is such that

$\operatorname{rank} D=l$

In order to investigate the robustness characteristics of the error-actuated digital PID controllers governed by equations (5), (6) and (7), Porter and Abidin [4] expressed the design equations for the controller matrices in equation (5) in the forms

$$
K_{1}=T \bar{H}^{-1} \Sigma\left(I_{l}+2 D\right)^{-1} \in R^{l x l}
$$

and

$$
K_{2}=\rho T \bar{H}^{-1}(T) \Sigma\left(T I_{l}+2 D\right)^{-1} e R^{l x l} .
$$

In equations (9) and (10),

$$
\Sigma=\sigma I_{l}\left(\sigma \in R^{+}\right)
$$

is the positive diagonal turning matrix,

$$
D=\delta I_{l}\left(\delta e R^{+}\right)
$$

is the positive diagonal derivative matrix, and $\rho \in R^{+}$ is the ratio of integral to proportional action. In addition,

$$
\bar{H}(T)=\int_{0}^{T} \bar{C} e^{\bar{A} t} \bar{B} d t e R^{2 x l}
$$

is the step-response matrix of the nominal plant with state-space triple $(\bar{A}, \bar{B}, \bar{C})$ which is used for design purposes in obtaining the controller for the actual plant with statespace triple $(A, B, C)$ governed by equations (1) and (2). The results thus obtained by Porter and Abidin [4] elucidate the required robustness characteristics of the digital PID controllers governed by equations $(5),(6)$ and (7).

However, these theoretical robustness results of Porter and Abidin [4] are restricted to the asymptotic case of fastsampling error-actuated digital PID controllers for which the sampling frequency $f=1 / T \rightarrow \infty$. Since it has so far proved impossible to solve theoretically the corresponding non-asymptotic problem, Porter and Allaoui [5] used genetic algorithms to solve the following version of the robustness problem:

In the case of finite sampling frequencies, determine the quadruple $\{\alpha, \sigma, \rho, \delta\}$ of controller design parameters such that optimal trajectory-tracking behaviour is obtained when a given robotic manipulator is controlled by a digital $P I D$ controller so as to track a given trajectory.

It is clear that the solution of this problem will provide an optimal quadruple $\{\alpha, \sigma, \rho, \delta\}$ of controller desigh parameters which is dependent upon the given manipulator, the given task, and the measure of trajectory-tracking performance used in the optimisation procedure.

In order to use genetic algorithms to solve this problem, Porter and Allaoui [5] showed that it is necessary only to encode the quadruple $\{\alpha, \sigma, \rho, \delta\}$ of controller design parameters in accordance with a system of concentrated, multi-parameter, mapped, fixed-point coding [1]. Thus, each quadruple $\{\alpha, \sigma, \rho, \delta\}$ of controller parameters is represented by a string of binary digits. Then, following any choice of 
an initial generation of such strings, successive generations of strings are obtained using the basic genetic operations of selection, crossover, and mutation [1]. These operations ensure that the successive generations of error-actuated digital PID controllers thus produced by the genetic algorithm tend to exhibit improving trajectorytracking performance in respect of any measure of the quality of such performance specified by the designer for any given robotic manipulator.

\section{ILLUSTRATIVE EXAMPLE}

This general approach to genetic robustification can be conveniently illustrated by designing trajectory-tracking digital PID controllers when various different performance measures are used in the case of the typical three-degree-of-freedom robotic manipulator previously investigated by Porter and Allaoui [5]. In this case, the manipulator is governed on $T e[0,+\infty)$ by state and output equations of the respective forms

and

$$
M(\theta) \ddot{\theta}+h(\theta, \dot{\theta})+g(\theta)=u
$$

$$
y=f(\theta)
$$

where $\theta \in R^{3}$ is the vector of joint angles, $u \in R^{3}$ is the vector of joint torques, $y \in R^{3}$ is the positional vector of the end effector in Cartesian space, $M(\theta) e R^{3 \times 3}$ is the inertia matrix, $h(\theta, \dot{\theta}) \propto R^{3}$ is the vector of centrifugal and Coriolis torques, $f(\theta) \in R^{3}$ is the vector of direct kinematic relationships, and $g(\theta) \propto R^{3}$ is the vector of gravitational torques. The numerical values of the inertial and kinematic parameters for a typical three-degree-offreedom robotic manipulator are given by Petropoulakis [3].

In the neighbourhood of any operating point in task space, this manipulator is a completely irregular sixth-order linear multivariable plant with three inputs and three outputs. In order to illustrate the genetic design procedure, it is instructive to design trajectory-tracking digital PID controllers for this robotic manipulator when undertaking the same task as that investigated by Porter and Allaoui [5]. Thus, consider that it is desired to design a controller for the linearised dynamics of this manipulator corresponding the end-effector position $10,0.45,0 / \mathrm{m}$. It is intended that this controller be used to cause the end effector of the robotic manipulator to track straight-line trajectories between the following points:

$\begin{array}{ll}I & (-0.5,0,-0.2) \mathrm{m} \\ \text { II } & (-0.4,0.3,0) \mathrm{m}, \\ \text { III } & (0.3,0.3,0.3) \mathrm{m}, \\ \text { IV } & (0.3,0.3,0.3) \mathrm{m}, \\ V & (-0.45,0.35,0) \mathrm{m}, \\ \text { VI } & (-0.45,0.35,0) \mathrm{m}\end{array}$

These transitions are effected with 'trapezoidal' acceleration, cruise, and deceleration profiles in the following times:

$\begin{array}{llll}I & \rightarrow & I I & 1.5 \mathrm{~s}, \\ I I & \rightarrow & I I & 2 \mathrm{~s}, \\ I I I & \rightarrow & I V & 0.5 \mathrm{~s}, \\ I V & \rightarrow & V & 2.5 \mathrm{~s}, \\ V & \rightarrow & V I & 0.5 \mathrm{~s},\end{array}$

In addition, after the initial transition $I \rightarrow I$, the manipulator grasps an additional payload of $5 \mathrm{~kg}$. It is evident that, because of the intrinsic non-linearity of the robotic manipulator and the sudden variation in payload, this tracking task constitutes a formidable test of robustness for the digital PID controller.

The genetic design of this controller for this trajectory-tracking task can be readily undertaken by minimising any measure of tracking performance specified by the designer. Thus, for example, consider the cost function

where

$$
\Gamma=\lambda_{1} \Gamma_{1}+\lambda_{2} \Gamma_{2}+\lambda_{3} \Gamma_{3}
$$

$$
\begin{aligned}
& \Gamma_{1}=\int_{0}^{\tau}\|e(t)\| d t \\
& \Gamma_{2}=\int_{0}^{\tau}\|\Delta e(t)\| d t \\
& \Gamma_{3}=\int_{0}^{\tau}\|\Delta u(t)\| d t
\end{aligned}
$$

and $\lambda_{1}, \lambda_{2}, \lambda_{3}$ are non-negative weighting parameters. In equations, (16), |17| and (18), $\tau$ is the duration of the tracking task, $e(t) \in R^{3}$ is the trajectory-tracking error vector in Cartesian space, $\Delta e(t) e R^{3}$ is the change in the error vector $e(t) \in R^{3}$ over a sampling period, $\Delta u(t) e R^{3}$ is the change in the control vector $u(t) e R^{3}$ over a sampling period, and $|$. denotes the Euclidean norm. The special case of genetic design considered by Porter and Allaoui [5] was that corresponding to the 
cost function, $\Gamma$, in equation (15) with $\lambda_{1}=1, \lambda_{2}=0$, and $\lambda_{3}=0$. However, the results of performing the genetic robustification procedure over 50 generations in more general cases are shown in Tables 1 and 2 for a population size $N=30$, a crossover probability $p_{c}=0.6$, and a mutation probability $p_{m}=0.005$. Thus, the results obtained by genetically minimising $\Gamma$ with $\lambda_{1}=1$ and $\lambda_{3}=0$ for different values of $\lambda_{2}$ are shown in Table $1_{i}$ whilst the corresponding results obtained by genetically minimising $\Gamma$ with $\lambda_{1}=1$ and $\lambda_{2}=0$ for different values of $\lambda_{3}$ are shown in Table 2 . It is evident from Table 1 that, as $\lambda_{2}$ increases, the value of $\Gamma_{2}$ decreases while the value of $\Gamma_{1}$ increases: the associated 'smoothing' of the trajectory-tracking behaviour of the robotic manipulator is demonstrated by comparing the optimised time-domain behaviour of $\|e(t)\|$ corresponding to $\lambda_{1}=1, \lambda_{2}=0$ and $\lambda_{3}=0$ shown in Figure 1 with that corresponding to $\lambda_{1}=1, \lambda_{2}=5 \times 10^{4}$, and $\lambda_{3}=0$ shown in Figure 2. Similarly, it is evident from Table 2 that, as $\lambda_{3}$ increases, the value of $\Gamma_{3}$ decreases while the value of $\Gamma_{1}$ increases: the associated 'smoothing' of the trajectorytracking behaviour of the manipulator is demonstrated by comparing the optimised time-domain behaviour of $\| e(t) \mid$ corresponding to $\lambda_{1}=1, \lambda_{2}=0$ and $\lambda_{3}=0$ shown in Figure 1 with that corresponding to $\lambda_{1}=1, \lambda_{2}=0$ and $\lambda_{3}=0.01$ shown in Figure 3 . It also transpires that the joint torques generated by the digital PID controllers are much 'smoother' in the case of the time-domain responses shown in Figures 2 and 3 than in the case of that shown in Figure 1. Finally, it is important to note from Tables 1 and 2 that the quadruples $\{\alpha, \sigma, \rho, \delta\}$ of optimal controller parameters corresponding to these quite different timedomain characteristics frequently do not differ from each other very much land yet the genetic algorithm has no difficulty in discriminating between these quadruples).

\section{CONCLUSION}

In this paper, genetic algorithms have been used to design digital multivariable PID controllers for robotic manipulators for typical trajectory - tracking tasks when various different performance measures are used. It is thus shown that, by using an appropriate performance measure, the set of controller design parameters can be readily found that determines the optimal time-domain trajectory-tracking behaviour for such tasks (which include sudden changes of payload). This use of genetic algorithms has been illustrated by the design of digital trajectorytracking PID controllers for the typical threedegree-of-freedom robotic manipulator previously investigated by Porter and Allaoui [5].

\section{REFERENCES}

[1] DE Goldberg, "Genetic Algorithms in Search, Optimization and Machine Learning", Addison-Wesley, 1989.

[2] J H Holland, "Adaptation in Natural and Artificial Systems", University of Michigan Press, 1975.

[3] L Petropoulakis, "Design of digital trajectory-tracking systems for robotic manipulators", PhD Thesis, University of Salford, 1986.

[4] B Porter and $Z$ Abidin, "Robustness characteristics of fast-sampling digital set-point tracking PID controllers for completely irregular linear multivariable plants", Proc 4th Inst MC Symposium on Application of Multivariable System Techniques, Bradford, England, April 1990.

[5] B Porter and C Allaoui, "Genetic robustification of digital trajectorytracking controllers for robotic manipulators", IEEE International Conference on Systems, Man and Cybernetics, Vancouver, Canada, October 1995.

[6] B Porter and A H Jones, "Genetic tuning of digital PID controllers", Electronics Letters, vol 28, p 843, 1992.

[7] B Porter, A Manganas, and T Manganas, "Design of fast noninteracting digital flight-mode control systems for high-performance aircraft", Proc AIAA Guidance, Navigation, and Control Conference, Snowmass, USA, August 1985.

[8] B Porter, S S Mohamed, and A H Jones, "Genetic tuning of digital PID controllers for linear multivariable plants", Proc. European Control Conference, Groningen, The Netherlands, June/July 1993. 


\begin{tabular}{|c|c|c|c|c|c|c|c|c||}
\hline$\lambda_{2}$ & $\Gamma$ & $\Gamma_{1}$ & $\Gamma_{2}$ & $\Gamma_{3}$ & $\alpha$ & $\sigma$ & $\rho$ & $\delta$ \\
\hline 0 & $1.399 \times 10^{-3}$ & $1.399 \times 10^{-3}$ & $1.201 \times 10^{-7}$ & 4.101 & 0.1104 & 0.8640 & 17.6337 & $1.280 \times 10^{-2}$ \\
\hline $10^{3}$ & $1.571 \times 10^{-3}$ & $1.466 \times 10^{-3}$ & $1.052 \times 10^{-7}$ & 2.411 & 0.1057 & 0.8786 & 18.2956 & $1.990 \times 10^{-2}$ \\
\hline $2 \times 10^{3}$ & $1.700 \times 10^{-3}$ & $1.535 \times 10^{-3}$ & $8.255 \times 10^{-8}$ & 2.410 & 0.0862 & 0.8568 & 18.6693 & $2.134 \times 10^{-2}$ \\
\hline $3 \times 10^{3}$ & $1.729 \times 10^{-3}$ & $1.577 \times 10^{-3}$ & $5.067 \times 10^{-8}$ & 2.26 & 0.1045 & 0.8832 & 19.9679 & $2.553 \times 10^{-2}$ \\
\hline $6 \times 10^{3}$ & $1.863 \times 10^{-3}$ & $1.863 \times 10^{-3}$ & $4.01 \times 10^{-8}$ & 2.07 & 0.1092 & 0.8810 & 18.2146 & $2.377 \times 10^{-2}$ \\
\hline $5 \times 10^{4}$ & $2.161 \times 10^{-2}$ & $1.949 \times 10^{-3}$ & $4.24 \times 10^{-9}$ & 2.44 & 0.0951 & 0.8870 & 11.4397 & $1.783 \times 10^{-2}$ \\
\hline
\end{tabular}

Table 1 : Genetic designs $\left(\lambda_{1}=1, \lambda_{3}=0\right)$

\begin{tabular}{|c|c|c|c|c|c|c|c|c||}
\hline$\lambda_{3}$ & $\Gamma$ & $\Gamma_{1}$ & $\Gamma_{2}$ & $\Gamma_{3}$ & $\alpha$ & $\sigma$ & $\rho$ & $\delta$ \\
\hline 0 & $1.399 \times 10^{-3}$ & $1.399 \times 10^{-3}$ & $1.201 \times 10^{-7}$ & 4.100 & 0.1104 & 0.8640 & 17.6337 & $1.280 \times 10^{-2}$ \\
\hline $10^{-3}$ & $3.673 \times 10^{-3}$ & $1.759 \times 10^{-3}$ & $1.088 \times 10^{-7}$ & 1.878 & 0.1485 & 0.8319 & 10.7541 & $1.235 \times 10^{-2}$ \\
\hline $5 \times 10^{-3}$ & $9.985 \times 10^{-3}$ & $2.280 \times 10^{-3}$ & $5.660 \times 10^{-3}$ & 1.541 & 0.1493 & 0.8517 & 15.9273 & $3.038 \times 10^{-2}$ \\
\hline $10^{-2}$ & $17.67 \times 10^{-3}$ & $2.616 \times 10^{-3}$ & $4.440 \times 10^{-3}$ & 1.505 & 0.1343 & 0.8528 & 13.3393 & $2.946 \times 10^{-2}$ \\
\hline $10^{-1}$ & 0.1389 & $7.784 \times 10^{-3}$ & $4.250 \times 10^{-7}$ & 1.311 & 0.1248 & 0.7321 & 10.4856 & $7.072 \times 10^{-2}$ \\
\hline 1 & 1.305 & $1.753 \times 10^{-2}$ & $2.592 \times 10^{-6}$ & 1.287 & 0.1142 & 0.6997 & 6.0821 & 0.1016 \\
\hline
\end{tabular}

Table 2 : Genetic designs $\left(\lambda_{1}=1, \lambda_{2}=0\right)$ 


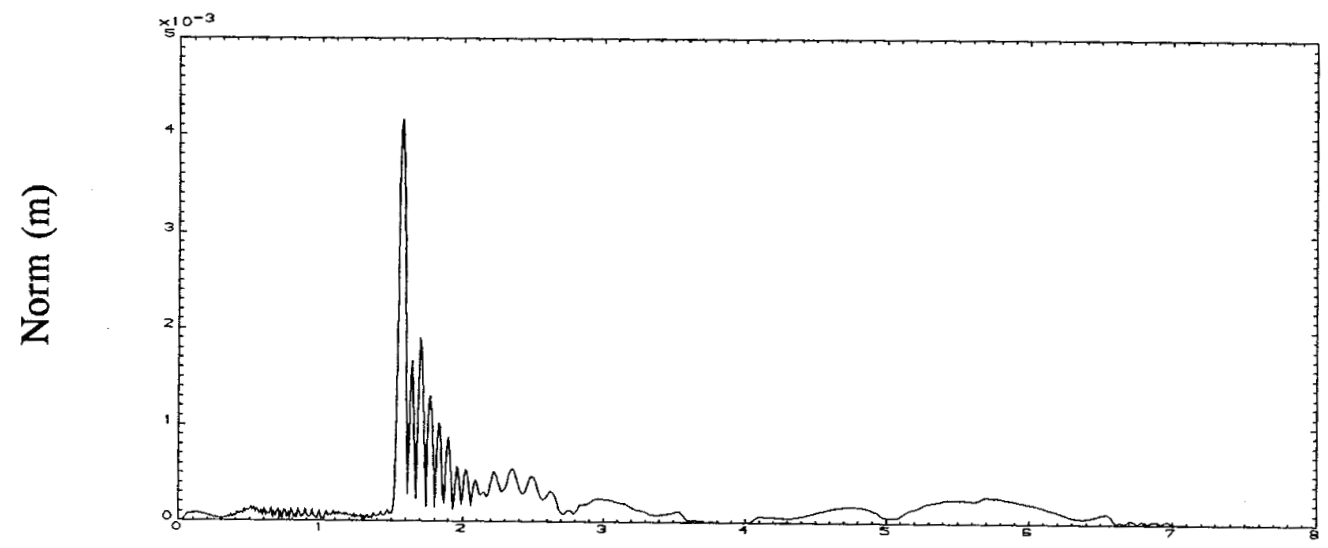

Time (s)

Fig 1 : Time-domain behaviour $\left(\lambda_{1}=1, \lambda_{2}=0, \lambda_{3}=0\right)$

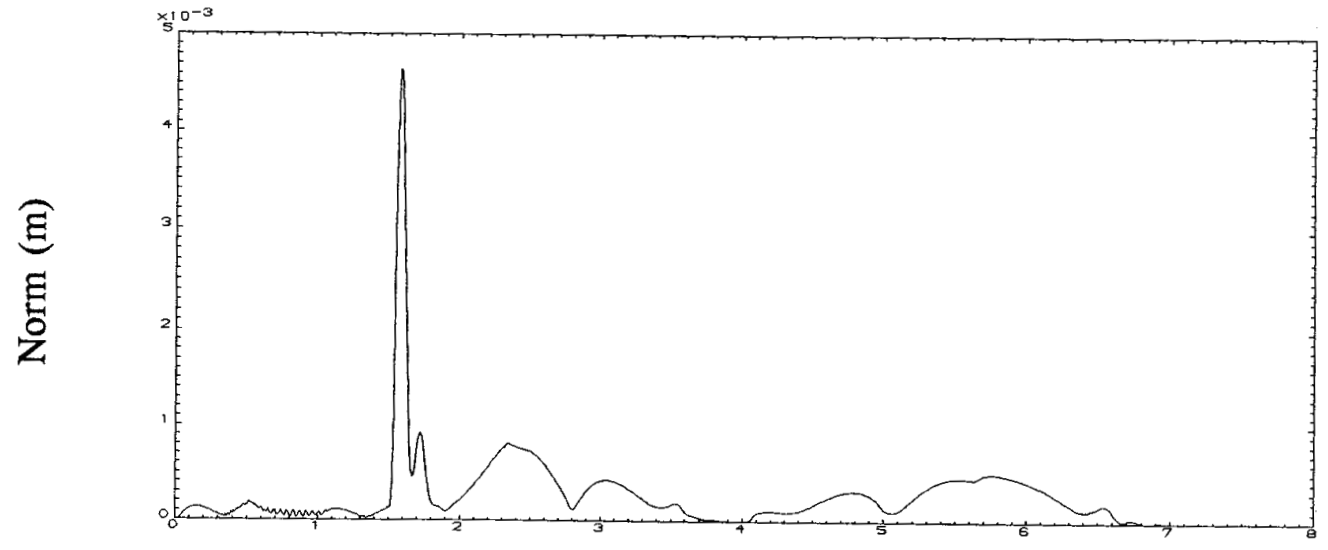

Time (s)

Fig 2 : Time-domain behaviour $\left(\lambda_{1}=1, \lambda_{2}=5 \times 10^{4}, \lambda_{3}=0\right)$

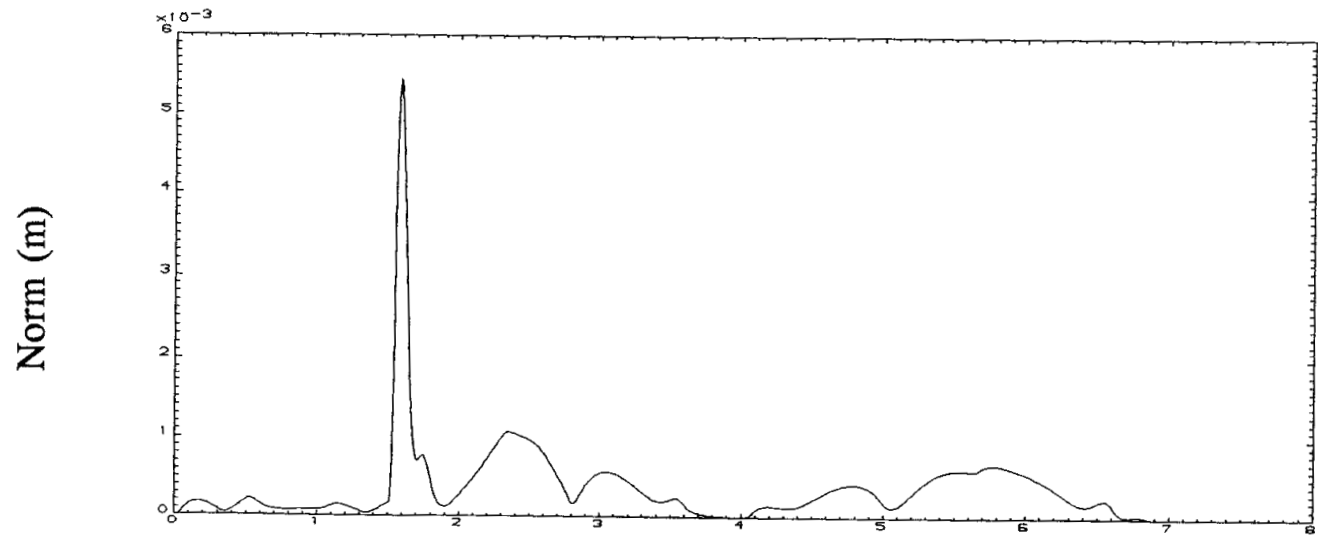

Time (s)

Fig 3 : Time-domain behaviour $\left(\lambda_{1}=1, \lambda_{2}=0, \lambda_{3}=0.01\right)$ 